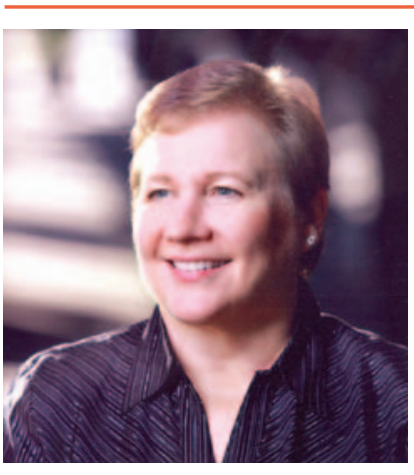

Lillie D. Shockney, RN, BS, MAS

Lillie D. Shockney, RN, BS, MAS is University Distinguished Service Associate Professor of Breast Cancer in the Departments of Surgery and Oncology, and Administrative Director of the Johns Hopkins Breast Center and the Johns Hopkins Cancer Survivorship Programs. She is Associate Professor at the Johns Hopkins University School of Medicine, in the Departments of Surgery, Oncology, and Gynecology and Obstetrics and Associate Professor at the Johns Hopkins University School of Nursing.
The ideas and viewpoints expressed in this editorial are those of the author and do not necessarily represent any policy, position, or program of NCCN.

\section{Perspectives on Surveillance and Survivorship: When to Make the Transition}

\author{
Lillie D. Shockney, RN, BS, MAS
}

The publication of the 2006 Institute of Medicine (IOM) report, From Cancer Patient to Cancer Survivor: Lost in Transition, brought a new challenge for cancer centers and oncology specialists across the country. ${ }^{1}$ The report strongly recommended that, at completion of cancer treatment, clinicians provide their patients with a summary of the treatment they received and details about the type of cancer they had. The report also recommended providing a detailed plan of care to be used going forward that includes follow-up appointments, tests, and recommendations for a healthier lifestyle, with the goal of reducing cancer recurrence or a new cancer.

The rationale for this recommendation is to help ensure a successful transition from patient to survivor through informing patients about the treatment they have received, what they need to do going forward regarding their cancer diagnosis and treatment, and who will provide their follow-up care. ${ }^{2}$ The catalyst for the report was the acknowledgment that cancer service needs will continue to grow ( $48 \%$ by 2020) because of an increasing number of patients with newly diagnosed cancer and cancer survivors (13.7 million in 2012, projected to be 22.0 million in 2020). At the same time, experts predict that the number of doctors practicing oncology will only increase by $14 \%{ }^{3,4}$ Therefore, oncologists will not be able to maintain long-term follow-up for the patients they have treated.

The report defined the posttreatment period as an explicit phase of the patient's experience with cancer. However, the specific timing of the transition was left to the discretion of the treating physicians. Debate and concern continue among oncologists and primary care physicians (PCPs) regarding the ability to operationally implement this transition process. Elements to be worked out include the need for detailed medical documents and a relatively lengthy consultation with the patient to review these documents; educating PCPs on their newly expanded role is as it relates to providing cancer survivorship care; and whether PCPs can expect cancer survivors to perform any self-monitoring. ${ }^{5,6}$

\section{Concerns and Potential Benefits}

Even if the process of creating these documents was simple, and communicating the information to patients was time-efficient, determining the ideal time to make this transition from the oncology team to the PCP presents a large challenge, because several must be considered.

\section{Bonding}

Patients often form bonds with members of their oncology team (ie, surgical oncologist, medical oncologist, oncology nurses, nurse navigators), and may see these providers as having saved their lives. Most patients do not want to transition if it means not seeing their oncology team anymore. As part of the research efforts at Johns Hopkins, we conducted focus groups involving breast cancer survivors and-in separate groupsoncologists and PCPs. Findings showed that patients are truly wedded to the providers they feel are the most skilled in continuing to provide long-term care. One patient claimed that she doesn't even feel the need for an examination; if her oncologist tells 
her that he can see she is doing well, then she believes she is and at that moment is cancer-free.

Oncologists expressed concern about feelings of loss, too, stating that they will miss seeing their patients, and that these types of follow-up visits are one of the things that gives them joy in their work. If long-term survivors were eliminated from their clinic schedules and replaced with more patients with newly diagnosed breast cancer, they feared they would experience burnout with their job. ${ }^{7}$ PCPs who were internal medicine specialists or generalists also expressed concern that they lack the time to effectively provide prevention and treatment of acute health issues and chronic disease management (eg, diabetes, heart disease, asthma, arthritis). They were also concerned that they have not been trained in long-term survivorship care.

An article on the time constraints of PCPs echoes this problem. ${ }^{7}$ On average, a PCP has 16 minutes to spend with a patient experiencing an acute illness. Once the presenting illness is addressed and possibly a few chronic comorbidities are assessed, the time remaining for prevention and survivorship care is 1 minute. ${ }^{8}$ Also of concern to PCPs is that they may lack confidence that they know all of the survivorship care issues that should be monitored and addressed, and therefore may default to performing a staging workup each time they see a patient to ensure no recurrence has occurred.

\section{Fear of Recurrence}

Fear of recurrence can also drive the patient to insist on receiving a staging workup (this was also noted in the focus group results). If this were to occur on a routine basis, the country's health care costs would be even more significant, because the average staging workup costs around $\$ 11,000$.

The survivorship care plan should contain information for the patient and any physician, including oncologists, cardiologists, and PCPs, regarding early identification of late effects and appropriate interventions to decrease physical symptoms and improve functional status. Conversely, highlighting all possible long-term or late effects may actually increase the patient's distress. Therefore, the survivorship care plan may improve perceived health and self-esteem for some patients, whereas for others it may seem overly focused on their previous cancer experience, increasing their fear of recurrence. From a psychosocial perspective, these survivors may experience great difficulty reclaiming their lives and moving forward. ${ }^{9}$

\section{Late Effects and Surveillance}

The survivorship care plan should also contain a description of the possible clinical course. This information should include the estimated time frame during which acute toxicities are expected to subside, a description of the long-term side effects not expected to improve much, and a suggestion regarding when to anticipate late effects.

For most cases, however, research on the incidence, risks, clinical characteristics, and outcomes of these effects is lacking. Additionally, as new treatments are implemented, longitudinal data may not be available to provide predictions about late effects and how to identify them. ${ }^{9}$ However, this does not mean we should ignore the late and long-term effects that we know about. For instance, we clearly know about cardiac late effects, and these should be closely followed.

\section{Gaps in Education}

The Survey of Physician Attitudes Regarding the Care of Cancer Survivors (SPARCCS) study revealed significant differences in the knowledge, attitudes, and 
practices of PCPs and oncologists in caring for cancer survivors. More than two-thirds of the physicians in the study departed significantly from clinical guidelines, with the primary problem being overuse of staging workup tests. ${ }^{10}$

Surveillance for recurrence is also a gray area. Recommendations regarding surveillance for cancer recurrence are unique to each type of cancer and its stage, the disease histology, and the presence of any suspected genetic predisposition. Thus, surveillance guidelines are clearly needed and must be communicated to the patient.

Another important point is that a small percentage of patients with certain cancers (eg, colon cancer, renal cell carcinoma, some sarcomas) that recur distantly with oligometastatic disease may be able to undergo surgery for a possible cure. ${ }^{11,12}$ In many clinical situations, however, intense monitoring of asymptomatic patients to identify distant metastases has no value, because palliative chemotherapy in these patients has not proven helpful in extending life or maintaining quality of life. ${ }^{9}$ This could be quite alarming for a patient to hear and difficult for them to understand.

NCCN has recently developed and published the NCCN Clinical Practice Guidelines in Oncology (NCCN Guidelines) for Survivorship (to view the most recent version of these guidelines, visit NCCN.org). Established guidelines for diseasespecific surveillance currently exist only for breast and colorectal cancer. NCCN is working on developing and implementing guidelines for other disease sites; however, these take time to develop, and more time to implement.

\section{Other Challenges}

Other challenges include the realization that the purpose of monitoring cancer survivors is to recognize potential problems related to their prior cancer treatment. An aging population, however, will develop comorbid conditions anyway, making it more difficult for a PCP to differentiate which condition is associated with cancer treatment and which may be the manifestation of a new primary acute or chronic disease. ${ }^{13}$

Another challenge is patient concern about receiving treatment from nonphysician advanced practitioners. Even when patients receive survivorship care at a cancer center, they may not like the idea of being seen by a physician extender (nurse practitioner or physician's assistant) rather than the doctors who treated their cancer.

Anecdotally, I have also heard of instances of "enabling" behavior during and after cancer treatment. For example, a patient with symptoms of a urinary tract infection (UTI) while undergoing chemotherapy calls her oncologist, who orders a urine culture, confirms the UTI, and calls in a prescription for an antibiotic. Three years after completion of her treatment, the patient calls the oncologist's office and says "I think my UTI is back." Rather than directing the patient to call the PCP, the oncologist might arrange for a urine culture and then prescribe an antibiotic. In that instance, or in similar situations, the cancer survivor considers the oncologist to be her new PCP.

Ensuring that survivors have access to good primary care may also be a challenge. The patient may not have had a PCP in the past, or may not have had a good relationship with the PCP. This may require negotiation for that individual to be seen as a new patient in an already overloaded practice, or for that patient to ask to be assigned to a different PCP. Building trust can be a challenge for both the physician and the patient.

In addition, the patient may seek solutions for symptoms associated with ongoing cancer treatment. For example, patients may request treatment for menopausal side effects caused by hormonal therapy used to prevent recurrence. I had a patient 
on tamoxifen who informed me that she felt much better after she had seen her gynecologist and received hormone replacement therapy (a class of drugs directly contraindicated for patients with breast cancer).

Another challenge to transition is that, while under the care of the oncology team, patients likely had access to many valuable resources, such as free transportation, food for their family, prescription coverage of certain drugs, other financial help, and even housecleaning services, that addressed a variety of barriers to care. These services are usually discontinued when patients are no longer seeing their oncologist. It is not practical for a nonprofit patient advocacy organization to continue these resources indefinitely. However, this change can mean financial or logistical problems for patients, and these problems can make it more difficult for them to adhere to certain instructions (such as taking cancer prevention drugs daily).

\section{Potential Benefits}

Transitioning can also provide some benefits. An area that PCPs are familiar with and manage in daily practice is the promotion of lifestyle changes that can prevent specific diseases, reduce the risk of worsening disease, and improve overall wellness. The end of acute cancer treatment has been called a "teachable moment." Patients may be more willing than ever before to adopt healthier PCP-recommended behaviors that they may have previously resisted. The fear of cancer recurrence can inspire patients to exercise, adopt a healthier diet, stop smoking, and get regular checkups. ${ }^{14}$

With the current fragmentation of medical care, it can be difficult for a patient or provider to know whom to contact for what. The survivorship care plan should help with this issue by explicitly defining which providers should do what, when, how, and why. This is especially important if the patient is still receiving some treatment or oversight by the oncologist while simultaneously re-engaging with their PCP for other medical care. In this case, the plan may serve as a documentation tool to prevent overuse or underuse of medical resources, such as 2 different providers ordering a mammogram for the same patient, or no one ordering a mammogram for that patient because everyone thought someone else had.

\section{Transition Needs}

To ensure the correct timing and success of the transition, expectations should be made clear at the first oncology consultation. Patients should be informed at that time how the process of treatment will occur and at what point they can expect to be transitioned back to their PCP. Patients should never be "surprised" during a consultation after their last acute cancer treatment by being informed that they will be migrating back to their PCP soon.

Ideally, transition timing should be based on prognostic factors and stage of disease, combined with the treatment that has been decided by the patient and oncology team. Although there are, of course, variations from this basic plan, the oncology team can project the period of time that risk of recurrence is of particular concern and when that risk is of less concern. For example, a breast cancer survivor who has undergone bilateral mastectomies to treat stage 0 breast cancer in one breast has a less than $1 \%$ risk of recurrence and should be transitioned in a matter of months to the PCP. Conversely, a patient who was diagnosed with and treated for stage III inflammatory breast cancer should remain under surveillance by the oncology team for 5 to 7 years because she has a high risk of local and distant recurrence.

Furthermore, early discussions between the oncology team and the patient should ideally include how both should maintain a relationship with the PCP. In this way, the 
patient is actually experiencing a shared-care survivorship model, meaning that the patient never totally disconnects from the PCP after the cancer diagnosis and during treatment. ${ }^{15} \mathrm{~A}$ shared-care model has also been validated to improve outcomes and facilitate effective management of chronic diseases. ${ }^{16}$

Lastly, a team of cancer survivors who had previously undergone the transition and are willing to serve as volunteer support for patients about to make the transition would also ideally be available. This would potentially address patients' emotional well-being more effectively. Doctors can tell patients "you will be fine." However, hearing "I was worried at first about leaving my oncology team but I understand why it's necessary, and I am building a better relationship with my PCP and taking steps to reduce my own risk, too. I am doing fine. You will, too" from a survivor who had the same type of cancer at the same stage and with the same prognostic factors is much more convincing.

Do such cancer survivors exist? Yes, they do. I am one of them. I am able to walk a patient with breast cancer through her treatment process and share my own story. I can make her aware that our goal is for her to eventually not need us anymore. That would be the best gift of all for her and for us: to not need the oncology team ever again.

\section{References}

1. Hewitt M, Greenfield S, Stovall E, eds. From Cancer Patient to Cancer Survivor: Lost in Transition. Washington, DC: National Academies Press; 2006.

2. Earle CC. Failing to plan is planning to fail: improving the quality of care with survivorship care plans. J Clin Oncol 2006;24:5112-5116.

3. Hortobagyi GN; American Society of Clinical Oncology. A shortage of oncologists? The American Society of Clinical Oncology workforce study. J Clin Oncol 2007;25:1468-1469.

4. Pratt-Chapman M, Trimble A, Leonard J, et al. Executive summary of cancer survivorship: a policy landscape analysis developed by the National Cancer Survivorship Resource Center. Available at: http://www.cancer.org/acs/groups/content/@editorial/documents/document/acspc-033812.pdf. Accessed September 25, 2013.

5. Cheung WY, Aziz N, Noone AM, et al. Physician preferences and attitudes regarding different models of cancer survivorship care: a comparison of primary care providers and oncologists. J Cancer Surviv 2013;7:343-354.

6. Virgo KS, Lerro CC, Klabunde CN, et al. Barriers to breast and colorectal cancer survivorship care: perceptions of primary care physicians and medical oncologists in the United States. J Clin Oncol 2013;31:2322-2336.

7. Kantsiper M, McDonald EL, Geller G, et al. Transitioning to breast cancer survivorship: perspectives of patients, cancer specialists, and primary care providers. J Gen Intern Med 2009;24(Suppl 2):S459-466.

8. Tai-Seale M, McGuire T, Zhang W. Time allocation in primary care office visits. Health Serv Res 2007;42:1871-1894.

9. Earle CC. Long term care planning for cancer survivors: a health services research agenda. J Cancer Surviv 2007;1:64-74.

10. Potosky A, Han OK, Rowland J, et al. Differences between primary care physicians' and oncologists' knowledge, attitudes, and practices regading the care of cancer survivors. J Gen Intern Med 2011;26:1403-1410.

11. Fong Y, Cohen AM, Fortner JG, et al. Liver resection for colorectal metastases. J Clin Oncol 1997;15:938-946.

12. Pfannschmidt J, Klode J, Muley T, et al. Pulmonary metastasectomy in patients with soft tissue sarcomas: experiences in 50 patients. Thorac Cardiovasc Surg 2006:54;489-492.

13. Oeffinfer KC, Mertens AC, Sklar CA, et al. Chronic health conditions in adult survivors of childhood cancer. N Engl J Med 2006;355:1572-1582.

14. Ganz PA. A teachable moment for oncologists: cancer survivors, 10 million strong and growing! J Clin Oncol 2005;23:5458-5460.

15. Oeffiner KC, McCabe MS. Models for delivering survivorship care. J Clin Oncol 2006;24:5117-5124.

16. Renders CM, Valk GD, de Sonnaville JJ, et al. Quality of care for patients with Type 2 diabetes mellitus-a long-term comparison of two quality improvement programmes in the Netherlands. Diabet Med 2003;20:846-852. 